\title{
A Review of Sensor Fusion Techniques for Underwater Vehicle Navigation
}

\author{
Tudor Nicosevici, Rafael Garcia, Marc Carreras and Miquel Villanueva \\ Computer Vision and Robotics Group \\ Institute of Informatics and Applications \\ University of Girona, E.P.S. \\ 17071 Girona, Spain \\ e-mail: \{tudor,rafa,marcc\}@eia.udg.es,miquel.villanueva@udg.es
}

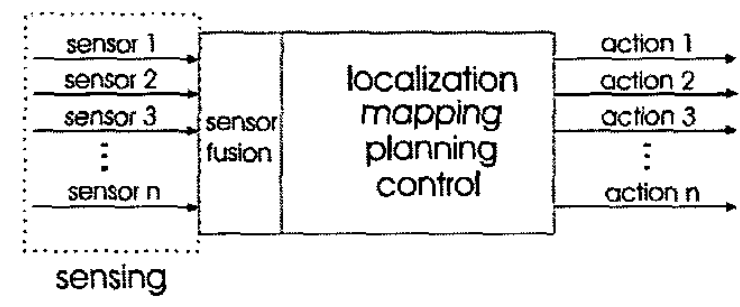

Fig. 1. Typical Navigation Architecture

\begin{abstract}
This paper provides a general description of the Multi Sensor Data Fusion concept, along with a new classification of currently used sensor fusion techniques for Unmanned Underwater Vehicles (UUV). Unlike previous proposals that focus the classification on the sensors involved in the fusion, we propose a synthetic approach that is focused on the techniques involved in the fusion and their applications in UUV navigation. We believe that our approach is better oriented towards the development of sensor fusion systems, since a sensor fusion architecture should be first of all focused on its goals and then on the fused sensors.
\end{abstract}

\section{INTRODUCTION}

Nowadays, when the mobile robots are used in a growing number of applications, the concept of robot navigation has been given a lot of attention. The term of navigation is quite generic (see Figure 1), since it includes aspects such as sensing, mapping, localization, planning, control, etc. In the case of exploratory missions, navigation becomes a difficult task, since there is no a-priori information of the environment where the mission takes place. This is the case of Unmanned Underwater Vehicles (UUV). In our previous work, the UUV was limited to navigate in structured environments [1] or survey small areas of the ocean floor [2] [3]. However, as UUVs normally carry out missions in wide, unstructured and highly dynamic environments, a reliable and "intelligent" navigation system is required.

A significant part of a robot navigation system is represented by the sensors carried by the robot. In the context of underwater navigation, a variety of sensors have been developed in the last years. The underwater navigation sensors can be classified in 5 general categories: GPS, Acoustic, Dead-Reckoning, Vision and Range-finders. The sensors of the first category, GPS based systems [4] offer a good navigation solution due to the absolute positioning capabilities. The drawback of this type of sensors is the necessity of the robot to rise to shallow depths in order to communicate with the satellite, being this a time and energy consuming task. On the other hand, acoustic systems [5] [6] [7] rely on external sound emitting beacons in order to triangulate its position. While precise, navigation based on this type of sensors is costly and limits the mission space to the area covered by the beacons.

Dead reckoning systems estimate the position of the UUV with respect to an initial point by measuring linear and angular velocities and/or accelerations (i.e. Inertial Navigation Systems, Doppler Velocity Logs etc.). Using this type of sensors offers a practical and inexpensive navigation method. Nevertheless, these sensors accumulate drifts over time since position errors tend to increase with each new measurement. Vision systems [8] allow the UUV to position itself with respect to the ocean floor by means of analyzing sequences of images provided by a downlooking camera. This approach follows the same principles as dead-reckoning techniques, with the addition of offering support for mapping applications. Finally, range-finder systems are usually used to provide supplementary data as the UUV cannot rely solely on them to navigate. The typical applications of these sensors are obstacle avoidance and local mapping.

Nevertheless, a reliable navigation system employs a sensor fusion module that improves the UUV state estimation by processing and merging the available 
sensory data. Section II overviews the sensor fusion principles. A new classification of sensor fusion techniques is proposed in section III. The classification is mainly oriented towards the goals that may be achieved using different fusion techniques (mapping, robot behaviour control, etc.). The paper concludes by offering a comparison among the discussed sensor fusion techniques.

\section{MULTI SENSOR DATA FUSION (MSDF)}

Multisensor integration can be defined as the synergistic use of the information provided by multiple sensory devices to assist the accomplishment of a task by a system [9].

Practically, multisensor fusion refers to any stage in the integration process where there is an actual combination (or fusion) of different sources of sensory information into one representational format. Explicitly, MSDF systems can fuse information from complementary sensors, redundant sensors or even from a single sensor over a period of time.

The fusion of sensor data provides a series of advantages in the context of UUV navigation: uncertainty can be reduced, noise can be rejected, sensor failure can be tolerated, resolution can be increased and the coverage of the sensors can be extended.

Depending on sensors and the goals of the MSDF, the sensor fusion can take place at different levels. Luo et al. [10] divide the MSDF into four levels: signal, pixel, feature and symbol. Signal level fusion decreases the covariance of the sensory data. Pixel-level fusion is intended to increase the information content associated to each pixel of an image. Feature-level fusion combines features derived from signals or images into meaningful representations or more reliable features. Symbol-level fusion allows the information to be fused at the highest level of abstraction and it is usually used in decision-based systems.

In practice, using similar sensors, it is sufficient to apply only one level of fusion. In more complex cases, when different groups of sensors are involved, each group is fused at a lower level, and the sensory data coming from different groups is fused at a higher level into one representational format. There are other cases, for instance when the fusion is behaviour-oriented, where the data coming from one sensor is fused at different levels, depending on the complexity of the behaviour. The levels involved in a fusion system and how are they organized define the type of data fusion architecture.

Hall et al. [11] define three types of MSDF architectures: centralized, autonomous and hybrid architectures. Centralized architectures deal with the fusion of raw observational data. Each of the sensorial information is aligned separately, transforming the units and coordinates of the sensor into the internal representation of the system. The data are then correlated to determine which sensorial information belongs to specific features of the environment. In autonomous architectures are distributed architectures, in which each sensor performs like a single-source state estimator. These estimates of position, velocity or acceleration are then joined together by means of a fusion system in order to achieve single state vector estimation. Finally, hybrid architectures combine data level fusion with state vector fusion. Therefore, each sensor can be either fused at data level or at vector level, depending on the demands of the system.

\section{A CLASSIFICATION OF MSDF TECHNIQUES}

This section outlines the most currently used MSDF techniques by discussing a series of proposals of sensor fusion-based navigation systems. The techniques have been classified into four main categories, based on the applications of the sensor fusion techniques: filtering and estimation, mapping-oriented, behaviour-oriented and machine learning.

The most basic MSDF systems are based on filtering and estimation techniques. Their purpose is usually limited to the estimation of the state of the vehicle. On the other hand, in cases of more complex navigation system, mapping-oriented techniques are more suited as they allow the fusion of sensory data at feature level, thus giving support for a more synthetic "understanding" of the environment.

Behaviour-oriented fusion systems are appropriate for instance for high-complexity missions, where it is more convenient to model the conduct of the vehicle as an interacting set of sub-tasks. Each of the sub-tasks is determined by one or more (fused) sensors and all the subtasks are fused together determining the overall vehicle behaviour.

Finally, machine-learning are implemented for complex navigation architectures, where manual modelling of the components becomes a difficult task.

\section{A. Filtering and estimation}

McGhee et al. [12] describe a navigation system employed by the Phoenix AUV using inertial and DGPS sensors. The sensory data is fused by means of a 9- state Kalman filter. The Kalman filter can be divided into seven continuous time states (three Euler angles, two horizontal velocities and two horizontal positions) and two discrete time states derived from the DGPS fixes. The main problem in the employed Kalman filter consists in the need of tuning the filter in order to avoid divergence. Doyle and Harris [13], Kobayashi et al. [14] and McGinnity and Irwin [15] propose a solution to this problem by using artificial intelligence techniques to overcome this problem.

Rendas et al. [16] propose a Kalman filter based fusion system. The sensory data is fused into variable dimension state vector. When there is no detectable acceleration, the system uses a model with uniform motion and linear 
velocity. When there is detectable acceleration, the system increases the system state vector in order to include the accelerations. The system fuses the data from an LBL positioning system and a dead reckoning system at signal level. When the AUV navigates within the area covered by the transponders, it positions itself using the LBL system. When outside the range of the transponders, the vehicle uses a combination of DVL, sonar and depth sensors for autonomous navigation. Practically, the AUV monitors the acoustic signals from beacons and when they become too weak, it automatically enters in dead reckoning navigation mode. When the vehicle is again able to receive the signals from the transponders, it switches back to LBL navigation. This proposal offers a good solution for AUV navigation since it combines the advantages of LBL and dead reckoning systems. Other authors also proposed the use Kalman based MSDF with variable size vectors. Drolet $e t$ al. [17] propose a sensor fusion algorithm that uses a bank of Kalman filters to represent different combinations of sensors. A model selection process selects the appropriate filters that represent the real system. This type of architecture offers tolerance to sensor failure and also offers the possibility of online-switching between different configurations of sensors depending on the AUV needs. Another important advantage of this approach is represented by the possibility to use asynchronous input from different sensors by switching between filters depending on the data availability.

Niwa et al. [18] also propose a Kalman based MSDF that accepts asynchronous input from sensors. The architecture consists in a vision sensor and an INS. As the vision sensor has lower data rate than the INS sensor, a Multi Rate Kalman Filter (MRKF) is employed to overcome this problem. When information is available from both sensors, the MRKF functions as a normal Kalman filter to estimate the state of the AUV. When only information from the INS is available, the filter assumes that the data from the vision sensor has infinite variance, forcing the filter to estimate the state of the AUV relying only on the INS data. This approach claims to be more flexible, also offering support to sensor failure tolerance. When a sensor is detected as defective, its variance is set to infinite, thus eliminating the erroneous data from the fusion process.

Zohdy et al. [19] propose a MSDF system that detects and eliminates erroneous sensory information when using multiple redundant sensors. The sensor is done by means of a band pass filter (BPF). The system creates a vector of the weighted averages of the sensor measurements. The band pass limits are chosen to be \pm 0.3 the deviation of the vector. If a sensor measurement lies outside the limits of the BPF, it is eliminated from the fusion process. As this method presents a simple and efficient way to tolerate sensor failures, it can prove to be expensive due to the necessity of multiple redundant sensors.

\section{$B$. Mapping-oriented}

In the context of Simultaneous Localization and Map Building (SLAM), most of the navigation architectures fuse the sensory information at feature level by projecting the features extracted from each of the sensor into a common state-space, referred to as map.

The main problem imposed by the SLAM navigation is the association between features extracted from different sensory sources (sensor alignment). This problem resides in different sensor properties, for example a vision sensor has good $x, y$ and angular resolution but provides no depth information; in contrast a sonar provides good depth information but has poor angular resolution. In order to overcome this problem, Neira et al. [20] propose the use of SPmodel, a probabilistic model to represent geometric environmental information. In this work, each geometric feature has a reference associated to it. The location of each feature reference with respect to the base reference is given by a vector transformation that in case of a 2D map is composed by two cartesian coordinates and an angle. Using this type of representation, a series of geometric associations among features is defined. In order to define the uncertainty in the location of a feature, a differential location vector is associated to the location vector. The vehicle navigation is done by means of specialized version of the extended Kalman filter that supports the SPmodel, referred to as the Spfilter.

Wei et al. [21] use an omnidirectional camera in conjunction with a ring of sonars for robot navigation. The system creates two parallel maps by projecting the information from each of the sensors into two different state-spaces. The two maps are then fused together to obtain a high-resolution occupancy grid representing the navigation environment.

A different approach was proposed by Majumder et al. [22] [23] [24]. They propose a multi-layered data fusion scheme to combine the information from sonar and underwater cameras into a complete environment map. All sensor information is projected into a common state-space before the extraction of seabed features. The feature extraction and subsequent processing is based on a combined description of the environment. Majumder et al. demonstrate that this approach is better then extracting the features from each of the sensors followed by fusion. In this work, two fusion approaches are discussed: extended Kalman filter (EKF) and Bayesian. The Bayesian approach is preferred as the EKF approach imposes two main problems: the difficulty of modelling natural environment features in a form accepted by the EKF and the fragility of the EKF when faced to incorrect associations of different observations.

An interesting proposal of an architecture suited for missions that involve manipulation tasks was made by Chantler et al. [25]. The system fuses information from sonar and laser triangulation devices, producing probabilistic 3D occupancy information only. The fusion architecture employs three main modules: the probabilistic 
sensor fusion module, the sensor error module and the interpretation control module. The output of each sensor undergoes some form of interpretation before being incorporated by the probabilistic sensor fusion module into a probabilistic volumetric model. The sensor error module defines how the raw sensor data is transformed into a standard probabilistic representation. The role of the interpretation control module is to expedite the interpretation of as requested by the planner levels in AUV architecture. Although simple and efficient, this architecture is not appropriate for large area navigation due to the lack of extraction of specific features of the environment.

Bison et al. [26] use a logical approach in the context of mapping and data fusion. Based on Possibilistic Logic [27], a descriptive language was developed that represent Dubois and Prade [28] [29] method of data fusion. The system uses a top-down segmentation system in order to classify the entities present in the environment using the information from each sensor. The sensory information is fused at symbol level using logic sentences.

\section{Behavior-oriented}

Unlike the classic Sensing-Modelling-Planning-Action navigation approach, the behavior-based approach consists of individual modules, each of them responsible for one behavior to be performed by the entire system [30] [31]. A module determines the specific behaviour by fusing information from one or more sensors connected to it. One sensor can be connected to one or more modules, thus influencing one or more behaviours [32]. The overall behaviour of the system is computed by fusing the specific behaviours.

Behaviour-based architectures usually employ two layers of sensor fusion: (i) module-layer sensor fusion where the sensory information is fused at signal, pixel or feature level and (ii) global-layer sensor fusion, where the sensorial information is indirectly fused by merging the specific behaviours at symbol level (i.e. obstacle avoidance, target tracking, trapping avoidance behaviours are fused, in order to determine the overall behaviour of the vehicle).

Kweon et al. [33] propose the use of three levels of behaviours for robot navigation: reflexive, adaptive and purposive level. All of the behaviours act as force vectors, which composed together determine the desired trajectory of the robot.

In the first case, the reflexive level integrates range measurement sensors, being responsible for obstacle avoidance. To ensure the safety of the vehicle, the response of the reflexive level has to be immediate. In order to do this, no planning or other high-level sensorial information processing is done. The output of this level is simply range forces from nearby obstacles acting as repulsive forces on the robot.

On the other hand, the adaptive level includes a series of different exploring behaviours: open-space explorer, free-space explorer, obstacle follower and so on. At this level a wide range of sensors are fused, usually at feature level, resulting in new information that helps to improve the world model from both qualitative and quantitative points of view. The forces generated at this level push the robot towards new and unexplored regions in the environment. Finally, the purposive level drives the robot towards the accomplishment of the mission. It fuses sensory information regarding target (objective) searching and tracking.

Behaviour-based architectures offer the advantage of decomposing the complex AUV navigation problem into components where specific fusion and estimation techniques can be used.

\section{Machine learning techniques}

In the context of complex and dynamic MSDF techniques, especially when different types of sensors are involved, traditional data association methods are difficult to implement. A series of authors have proposed systems that have the ability to construct their own rules for data association and fusion (referred to as Machine Learning Techniques). These techniques include: neural networks [34], support vector machines [35], neural networks, etc. Among the machine learning techniques, the most widely adopted is the use of neural networks (NN) due to their high learning and adaptability capabilities.

Though, NN suffer from an important drawback: they need extensively long training time. Many alternatives have been proposed in order to optimize the training process while maintaining the qualities of the $\mathrm{NN}$ (i.e. localized receptive fields [36], radial basis function [37]).

Bailey et al. [34] propose the use of a NN based MSDF system where the fusion is done at two levels: data level and decision level (also called symbol level). Redundant sensory information is fused at data level by means of a Gabor filter. The data level fusion is done in two stages: (1) computation of Gabor coefficients using a three layered adaptive network and (2) classifying the filter correspondences. Complementary sensory information is fused at decision level by comparing the network outputs from each sensor using logical reasoning, which is learnable in neural networks by supervised learning. A feed forward network with BP learning was employed to learn to fuse the decisions.

The association problem when fusing information provided from two unequal data-rate sensors (an INS and a Scene Matching Guidance system) was outlined by Jiang et al. [38]. The solution proposed by the authors involves the use of a Hopfield neural network (HNN). The optimal fusion is obtained by minimizing the energy function of the HNN.

Mukai et al. [39] use a polynomial learning method in order to overcome the data association problem between two sensors: a vision sensor and an acoustic positioning system. The authors describe the data association problem as being a result of sensor data contradiction due to 
difference in view points, aberration of lenses and distortion of sensor coordinates. In order to deal with this problem, a mapping from one sensor to the other is defined. The shape of the mapping is defined by a set of Legendre polynomials, whom parameters can be adjusted by learning.

\section{CONCLUSIONS}

The paper provides a new and more generic classification of the sensor fusion techniques, transparent to the sensor types involved in the MSDF.

Table I provides the main characteristics of the MSDF techniques presented in previous chapter. The fusion techniques are organized in the table, from the most basic to the most advanced.

More advanced MSDF techniques fuse the sensory data at higher level of abstraction, allowing the modelling of more complex environments. More basic fusion techniques require more precise sensory information (i.e. noise covariances), there are cases where this is a difficult task due to the complexity of the sensors. Another problem imposed by the basic MSDF techniques is the data alignment (sensor registration) problem. In the case of more advanced MSDF techniques this problem becomes less restrictive, as the data is fused at a more abstract level.

The overall conclusion that can be drawn is that when developing a sensor fusion system there are two main issues that have to be taken into account: $(i)$ the goal of the fusion (the improvement brought by a specific technique) and (ii) the constrains imposed by the sensors (sensors involved in the fusion, sensory data model etc.)

\section{REFERENCES}

[1] M. Carreras, P. Ridao, R. Garcia, and T. Nicosevici, "Vision-based localization of an underwater robot in a structured environment," in IEEE International Conference on Robotics and Automation (ICRA), 2003.

[2] X. C. R. Garcia, J. Batlle and J. Amat, "Positioning an underwater vehicle through image mosaicking," IEEE International Conference on Robotics and Automation (ICRA), vol. 3, pp. 2779-2784, 2001.

[3] R. Garcia, J. Puig, P. Ridao, and X. Cufi, "Augmented state Kalman filtering for auv navigation," IEEE International Conference on Robotics and Automation (ICRA), pp. 4010-4015, 2002.

[4] T. Moore, "An introduction to the global positioning system and its applications," Developments in the Use of Global Positioning Systems, pp. 1/1 -1/6, Feb 1994.

[5] D. Jourdan, and B. Brown, "Improved navigation system for USBL users," in IEEE OCEANS Conference (OCEANS), vol. 1, Oct 97, pp. 727-735.

[6] F. P. Parthiot, and J. F. Denis, "A better way to navigate on deep sea floors," in IEEE OCEANS Conference (OCEANS), vol. 2, Oct 1993, pp. 494498.

[7] A. Matos, N. Cruz, A. Martins, and F. L. Pereira, "Development and implementation of a low-cost lbl navigation system for an AUV," in IEEE OCEANS Conference (OCEANS), vol. 2, Sept. 1999, pp. 774 779.

[8] R. Garcia, X. Cufi, and M. Carreras, "Estimating the motion of an underwater robot from a monocular image sequence," in IEEE/RSJ International Conference on Intelligent Robots and Systems (IROS), 2001, pp. 1682-1687.

[9] R. C. Luo, and M. G. Kay, "A tutorial on multisensor integration and fusion," in $16^{\text {th }}$ Annual Conference of IEEE Industrial Electronics Society (IECON), vol. 1, Nov 1990, pp. 707-722.

[10] R. C. Luo, Y. Chih-Chen, and K. L. Su, "Multisensor fusion and integration: approaches, applications, and future research directions," IEEE Sensors Journal, vol. 2, no. 2, pp. 104-119, April 2002.

[11] D. L. Hall and J. Llinas, "An introduction to multisensor data fusion," in IEEE Sensors Journal, vol. 85, no. 1, Jan 1997, pp. 6-23.

[12] R. B. McGhee, J. R. Clynch, A. J. Healey, S. H. Kwak, D. P. Brutzman, X. P. Yun, N. A. Norton, R. H. Whalen, E. R. Bachmann, D. L. Gay, and W. R. Schubert, "An experimental study on an integrated GPS/INS system for shallow water AUV navigation," in International Symposium on Umanned Unthetered Submersible Techonology, 1995, pp. 153-167.

[13] R. S. Doyle and C. J. Harris, "Multisensor data fusion for helicopter guidance using neurofuzzy estimation algorithms," The Royal Aeronautical Society Journal, pp. 241-251, June 1996.

[14] K. Kobayashi, K. C. Cheok, K. Watanabe, and F. Munekata, "Accurate differential global positioning via fuzzy logic Kalman filter sesor fusion technuque," in IEEE Transaction on Industrial Electronics, vol. 3, 1998, pp. 510-518.

[15] S. McGinnity and G. Irwin, "Nonlinear state estimation using fuzzy local linear models," International Journal of Systems Science, vol. 7, pp. 643-656, 1997.

[16] M. J. Rendas and I. M. J. Lourtie, "Hybrid navigation system for long range navigation," in Symposium on Autonomous Underwater Vehicle Technology, 1994, pp. 353-359.

[17] L. Drolet, F. Michaud, and J. Cote, "Adaptable sensor fusion using multiple Kalman filters," in IEEE/RSJ International Conference on Intelligent Robots and Systems (IROS), vol. 2, Nov 2000, pp. 1434-1439.

[18] S. Niwa, T. Masuda, and Y. Sezaki, "Kalman filter with time-variable gain for a multisensor fusion system," in IEEE/SICE/RSJ International Conference on Multisensor Fusion and Integration for Intelligent Systems (MFI), Aug 1999, pp. 56-61.

[19] M. A. Zohdy, A. A. Khan, and P. Benedict, "Fused multi-sensor data using a Kalman filter modified with interval probability support," in American Control Conference, vol. 5, June 1995, pp. 3046-3049. 


\begin{tabular}{|c|c|c|c|c|}
\hline Characteristics & $\begin{array}{l}\text { Filtering and } \\
\text { Estimation }\end{array}$ & Mapping Oriented & Behaviour Oriented & Machine Learning \\
\hline $\begin{array}{l}\text { Sensor Fusion } \\
\text { Level }\end{array}$ & Signal Level & Feature Level & $\begin{array}{l}\text { Mult Level (Feature } \\
\text { and Symbol) }\end{array}$ & $\begin{array}{l}\text { Multi Level (Feature } \\
\text { and Symbol) }\end{array}$ \\
\hline $\begin{array}{l}\text { Sensory Data } \\
\text { Model }\end{array}$ & $\begin{array}{l}\text { Random Variables - } \\
\text { Uncorrelated Noise }\end{array}$ & $\begin{array}{l}\text { Orientations, Positions, } \\
\text { Geometric Relations }\end{array}$ & Vectors & $\begin{array}{l}\text { Probabilistic } \\
\text { Measures }\end{array}$ \\
\hline Fusion Methods & Signal Estimation & $\begin{array}{l}\text { Geometrical Corresp. } \\
\text { and Feature Fusion }\end{array}$ & Vector Operations & $\begin{array}{l}\text { Fuzzy Logic, Neural } \\
\text { Networks, etc. }\end{array}$ \\
\hline $\begin{array}{l}\text { Sensor } \\
\text { Registration }\end{array}$ & $\begin{array}{l}\text { High Spatial and } \\
\text { Temporal }\end{array}$ & $\begin{array}{l}\text { High-Medium Spatial, } \\
\text { Medium-Low Temporal }\end{array}$ & $\begin{array}{l}\text { Medium-Low Spatial } \\
\text { and Temporal }\end{array}$ & $\begin{array}{l}\text { Medium-Low Spatial } \\
\text { and Temporal }\end{array}$ \\
\hline Improvement & $\begin{array}{l}\text { Reduction of } \\
\text { Variances }\end{array}$ & $\begin{array}{l}\text { Increased Featiue } \\
\text { Accuracy and } \\
\text { Complexity }\end{array}$ & $\begin{array}{l}\text { Increased Bebaviour } \\
\text { Precision and } \\
\text { Reliability }\end{array}$ & $\begin{array}{l}\text { Improved } \\
\text { Probabilities and } \\
\text { Decisions }\end{array}$ \\
\hline
\end{tabular}

TABLE I

Multi Sensor Data Fusion Techniques - Main Characteristics

[20] J. Neira, J. Horn, J. D. Tardos, and G. Schmidt, "Multisensor mobile robot localization," IEEE International Conference on Robotics and Automation (ICRA), vol. 1, pp. 673-679, April 1996.

[21] S. C. Wei, Y. Yagi, and M. Yachida, "Online map building based on ultrasonic and image sensor," in IEEE International Conference on Systems, Man, and Cybernetics, vol. 2, Oct 1996, pp. $1601-1605$.

[22] S. Majumder, S. Sheding, and H. F. Durrant-Whyte, "Sensor fusion and map building localisation for underwater navigation," in International Symposium on Experimental Robotics, 2000.

[23] S. Majumder, S. Sheding, and H. F. Durrant-Whyte, "Sensor fusion and map building for underwater navigation," in Australian Conference on Robotics and Automation, 2000, pp. 25-30.

[24] S. Majumder, S. Sheding, and H. F. Durrant-Whyte, "Multisensor data fusion for underwater navigation," Robotics and Automation Systems, vol. 35, pp. 97$108,2001$.

[25] M. J. Chantler, D. B. Lindsay, C. S. Reid, and V. J. C. Wright, "Optical and acoustic range sensing for underwater robotics," in OCEANS Engineering for Today's Technology and Tomorrow's Preservation (OCEANS), vol. 1, Sept 1994, pp. 205-210.

[26] P. Bison, G. Chemello, C. Sossai, and G. Trainito, "Logic-based sensor fusion for localization," in IEEE International Symposium on Computational Intelligence in Robotics and Automation (CIRA), July 1997, pp. 254-259.

[27] D. Dubois and H. Prade, "A survey of belief revision and updating rules in various uncertainty models," International Journal of Intelligent Systems, vol. 9, pp. 61-100, 1994.

[28] D. Dubois and H. Prade, "Possibility theory and data fusion in poorly informed environments," Control Engeneering Practice, vol. 2, no. 5, pp. 811-823, 1994.
[29] D. Dubois and H. Prade, "Combination of Fuzzy Information in the Framework of Possibilty Theory," Academic Press, 1992.

[30] R. A. Brooks, "Intelligence without reason," Artificial Intelligence Lab., Tech. Rep., 1991.

[31] R. A. Brooks, "A robust layered control system for a mobile robot," IEEE Journal of Robotics and Automation, vol. 2, April 1986.

[32] C. M. Pearson and P. J. Probert, "A verified architecture for sensor fusion," in IEEE/RSJ International Workshop on Intelligent Robots and Systems (IROS), vol. 2, Nov 1991, pp. 1089-1094.

[33] I. Kweon, Y. Y. Kuno, M. Watanabe, and K. Onoguchi, "Behaviour-based mobile robot using active sensor fusion," in IEEE International Conference on Robotics and Automation (ICRA), vol. 2, 1992, pp. 1675-1682.

[34] G. Bailey, S. Raghavan, N. Gupta, B. Lambird, and D. Lavine, "Infuse-an integrated expert neural network for intelligent sensor fusion," in IEEE/ACM International Conference on Developing and Managing Expert System Programs, Sept 1991, pp. 196-201.

[35] V. Vapnik, "The Nature of Statistical Learning Theory," Springer-Verlag, 1995.

[36] J. Moody and C. Darken, "Fast learning in networks of locally tuned processing units," in Neural Computation, vol. 1, 1989, pp. 281-294.

[37] A. Saha and J. Keeler, "Algorithms for better representation and faster learning in radial basis function networks," Advances in Neural Information Processing System, vol. 2, pp. 482-489, 1989.

[38] C. Jiang and Z. Chen, "Multisensor fusion using Hopfield neural network in INS/SMGS integrated system," in International Conference on Signal Processing, vol. 2, 2002, pp. 1199-1202.

[39] T. Mukai, T. Mori, and M. Ishikawa, "A sensor fusion system using mapping leaming method," in IEEE/RSJ International Conference on Intelligent Robots and Systems (IROS), vol. 1, July 1993, pp. 391-396. 